\title{
Overview of jet physics results from ALICE
}

\author{
Filip $\mathrm{Krizek}^{1, *}$ for the ALICE Collaboration \\ ${ }^{1}$ Nuclear Physics Institute of CAS, Husinec-Rez, Czech Republic
}

\begin{abstract}
Recent results from jet shower-shape and substructure analyses done by the ALICE collaboration in central Pb-Pb collisions at $\sqrt{s_{\mathrm{NN}}}=2.76 \mathrm{TeV}$ and in pp collisions at $\sqrt{s}=7 \mathrm{TeV}$ are reviewed. The presented jet showershape observables are angularity $g$ and transverse momentum dispersion $p_{\mathrm{T}} D$, which were studied for a small resolution parameter $R=0.2$, track-based jets with a minimum constituent transverse momentum $\left(p_{\mathrm{T}}\right)$ cut-off of $0.15 \mathrm{GeV} / c$. Jet substructure is explored for track-based anti- $k_{\mathrm{T}}$ jets with $R=0.4$ by means of iterative declustering and grooming techniques, which were used to measure the absolutely-normalized leading subjet momentum fraction $z_{\mathrm{g}}$ and the number of hard splittings in the reclustered jet shower, $n_{\mathrm{SD}}$. These observables provide complementary information on the jet fragmentation and help to discriminate between different scenarios for medium-induced modifications of the parton shower in heavy-ion collisions due to jet quenching.
\end{abstract}

\section{Introduction}

Collisions of ultra-relativistic heavy ions are used to probe strongly interacting matter in the regime of high-energy densities and temperatures. In this regime, color confinement of quarks and gluons in hadrons breaks down and a new state of matter, called Quark-Gluon Plasma, is formed [1]. Properties of this medium can be inferred e.g. based on observed modifications of produced jets, collimated sprays of particles that emerge as a result of fragmentation of highly-virtual partons after an initial hard scattering [2]. Jet-medium interaction proceeds via collisional and radiative processes of the partonic shower inside the medium. As a result, we observe jet quenching [3], which consists in yield suppression of high- $p_{\mathrm{T}}$ hadrons and jets with a finite radius $R$. In addition, the jet-medium interaction also leads to jet substructure modifications which can be quantified by different jet-shape observables or identified as changes in shower evolution when unwinding jet clusterization history.

Jet-shape observables are constructed as functions of jet constituent four-momenta and they are designed to accentuate some characteristic feature of the shower. For instance, angularity helps to quantify a degree of jet collimation [4]. It is defined as

$$
g=\sum_{i \in \text { jet }} \frac{p_{\mathrm{T}, i}}{p_{\mathrm{T}, \mathrm{jet}}}\left|\Delta R_{\mathrm{jet}, i}\right|,
$$

where $\Delta R_{\mathrm{jet}, i}$ denotes the angle enclosed by the $i$-th jet constituent and the jet axis. The ratio $p_{\mathrm{T}, i} / p_{\mathrm{T}, \mathrm{jet}}$ then gives the fraction of jet transverse momentum which is carried by this

\footnotetext{
*e-mail: krizek@ujf.cas.cz
} 
constituent. Jets with small angularity are more collimated and in the limit of pencil-like jets, the angularity tends to zero.

Another jet-shape observable, called transverse momentum dispersion, quantifies the hardness of the jet fragmentation and it is related to dispersion of the constituent momenta [4],

$$
p_{\mathrm{T}} D=\frac{\sqrt{\sum_{i \in \mathrm{jet}} p_{\mathrm{T}, i}^{2}}}{\sum_{i \in \mathrm{jet}} p_{\mathrm{T}, i}} .
$$

Jets with a large number of soft constituents will have $p_{\mathrm{T}} D$ values closer to 0 whereas jets where just a few constituents carry a large fraction of jet momentum will tend to have $p_{\mathrm{T}} D$ values closer to 1 [4].

Jet substructure measurements analyze history of steps, in which a jet algorithm assembled jet constituents together. This information is accessible via iterative declustering which unwinds the sequence of jet branch mergings. In heavy-ion collisions, jets are usually reconstructed using the anti- $k_{\mathrm{T}}$ jet algorithm [5]. This algorithm has reduced susceptibility with respect to the soft underlying event but at the same time, it does not reflect important characteristics of a partonic shower, e.g., the angular ordering. Therefore, anti- $k_{\mathrm{T}}$ jet constituents are subsequently once again reclustered with the Cambridge-Aachen algorithm [2]. In order to identify hard splittings within the jet shower, one uses grooming techniques like the Soft Drop (SD) [6]. In the given clusterization step, there are two branches with transverse momenta $p_{\mathrm{T}, 1}$ and $p_{\mathrm{T}, 2}$. The SD grooming condition that is evaluated is

$$
z=\frac{\min \left(p_{\mathrm{T}, 1}, p_{\mathrm{T}, 2}\right)}{p_{\mathrm{T}, 1}+p_{\mathrm{T}, 2}}>z_{\mathrm{cut}} \theta^{\beta},
$$

where $z_{\text {cut }}$ is a chosen momentum-fraction cutoff, $\theta$ is an opening angle of the splitting and $\beta$ is a parameter. This article reports results for $\beta=0$ and $z_{\text {cut }}=0.1$, which allow us to select hard splittings in the jet shower. In the case that a given splitting fails the grooming condition, the harder branch of the splitting is followed and decomposed in the subsequent unwinding step. Two substructure observables will be discussed here. The first one, called $z_{\mathrm{g}}$, measures the momentum fraction of the first splitting that fulfills $z>0.1$. To leading order, the measurement of $z_{\mathrm{g}}$ in vacuum is closely related to the Altarelli-Parisi splitting functions [6]. Theoretical considerations about the in-medium modification of $z_{\mathrm{g}}$ can be found in [1215]. The second observable counts the number of splittings that fulfill $z>0.1$ when we follow the hardest branch and it will be denoted $n_{\mathrm{SD}}$.

\section{Results from jet shape measurements}

Recently, the ALICE collaboration reported measurement of angularity and $p_{\mathrm{T}} D$ in $\mathrm{Pb}-\mathrm{Pb}$ collisions at $\sqrt{s_{\mathrm{NN}}}=2.76 \mathrm{TeV}$ and in pp collisions at $\sqrt{s}=7 \mathrm{TeV}$ [4]. The analysis was performed for track-based, anti- $k_{\mathrm{T}}$ jets with a resolution parameter of $R=0.2$ and a minimum jet constituent transverse momentum cut-off of $150 \mathrm{MeV} / c$. The measured spectra corrected for instrumental and underlying event effects are shown in Fig. 1. The upper panels present angularity and $p_{\mathrm{T}} D$ spectra for jets with transverse momentum $40-60 \mathrm{GeV} / c$ measured in $\mathrm{pp}$ collisions at $\sqrt{s}=7 \mathrm{TeV}$. The spectra are well reproduced by simulated distributions generated by two different PYTHIA tunes [7]. The bottom panels show the corresponding comparison of jet-shape distributions for pp collisions at $\sqrt{s}=2.76 \mathrm{TeV}$ generated by PYTHIA and the fully corrected jet-shape spectra obtained in central $\mathrm{Pb}-\mathrm{Pb}$ collisions at $\sqrt{s_{\mathrm{NN}}}=2.76 \mathrm{TeV}$ ( $0-10 \%$ centrality bin). The comparison suggests that the measured $40-60 \mathrm{GeV} / c$ jets exhibit a shift toward lower angularity and greater $p_{\mathrm{T}} D$ values. Thus it can be seen that jet-medium 

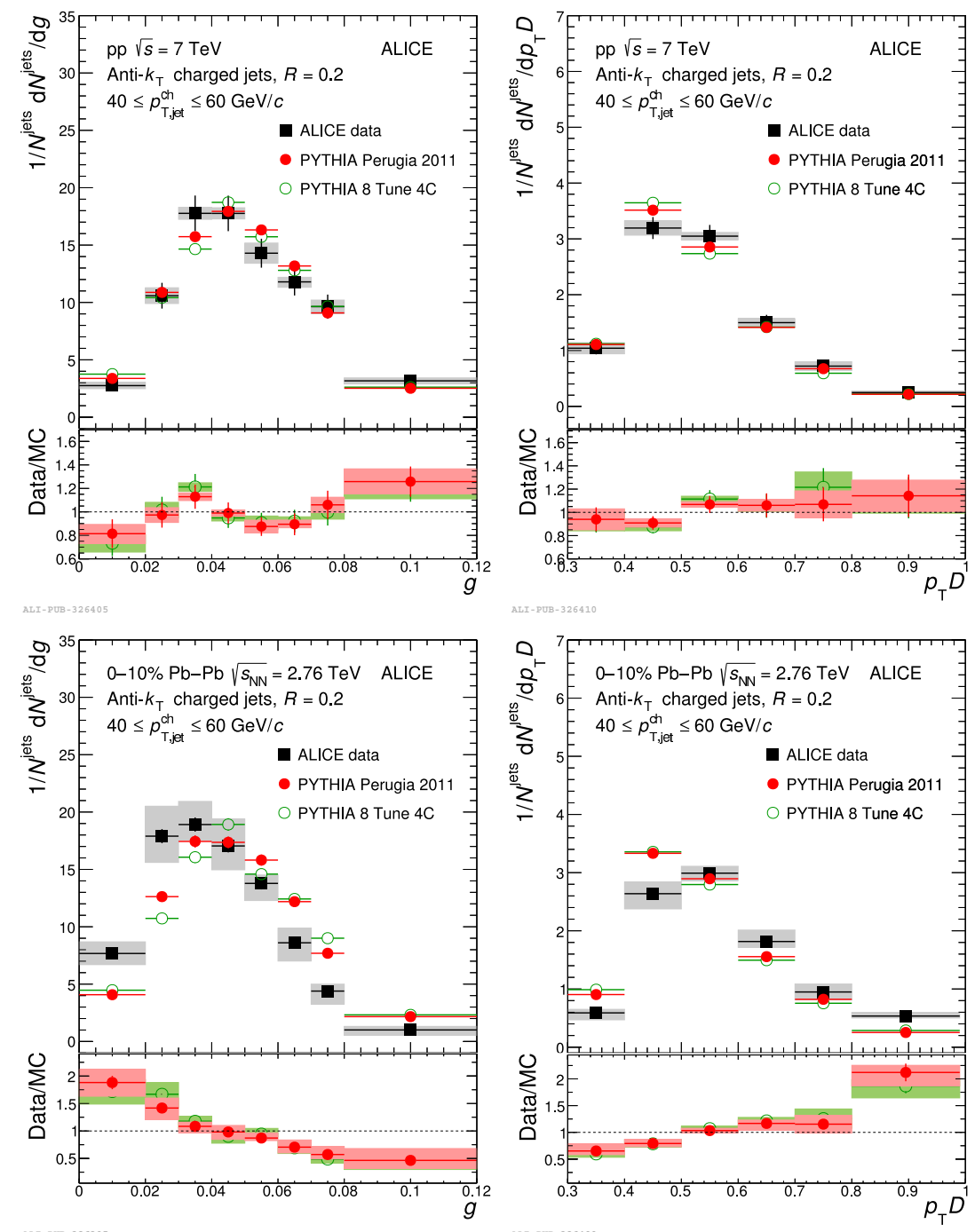

Figure 1. Fully corrected spectra of jet-shape observables as measured by ALICE in pp collisions at $\sqrt{s}=2.76 \mathrm{TeV}$ (top panels) and central $\mathrm{Pb}-\mathrm{Pb}$ collisions at $\sqrt{s_{\mathrm{NN}}}=2.76 \mathrm{TeV}$ (bottom panels) for track-based anti- $k_{\mathrm{T}}$ jets with $R=0.2$. Transverse momentum of the jets is constrained to the range $40-60 \mathrm{GeV} / c$. The left panels show angularity and the right panels present $p_{\mathrm{T}} D$ distributions. Data are compared with predictions of the PYTHIA event generator. See text for further details. Taken from [4].

interaction led to noticeable collimation of these $R=0.2$ jets and at the same time resulted also in more asymmetric fragmentation when compared to the vacuum expectation represented here by the PYTHIA simulation. Both features are qualitatively consistent with more quark-like fragmentation [4]. 

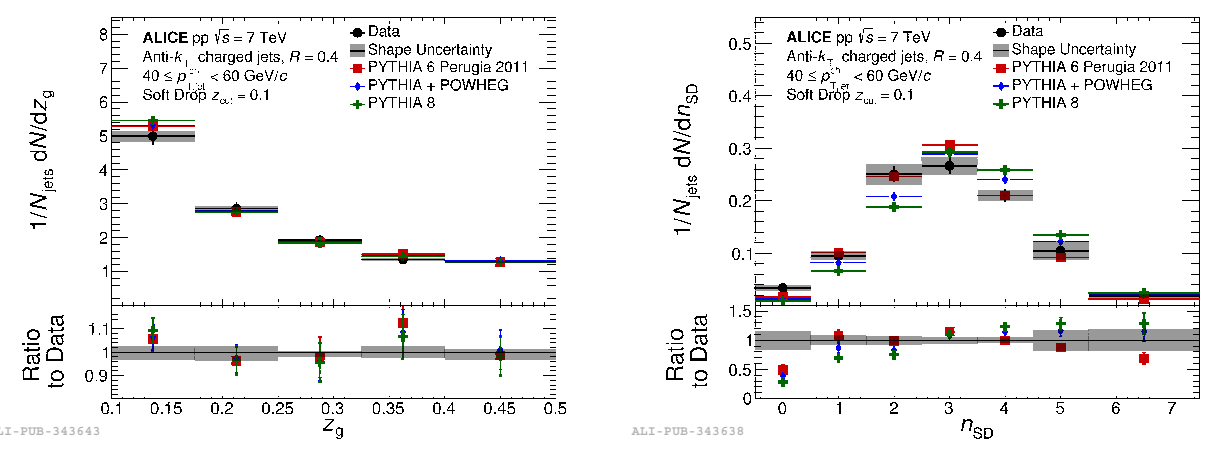

Figure 2. Fully corrected distributions of jet substructure observables $z_{\mathrm{g}}$ and $n_{\mathrm{SD}}$ as measured in pp collisions at $\sqrt{s}=7 \mathrm{TeV}$. Data are compared with PYTHIA event generator calculations. Taken from [8].

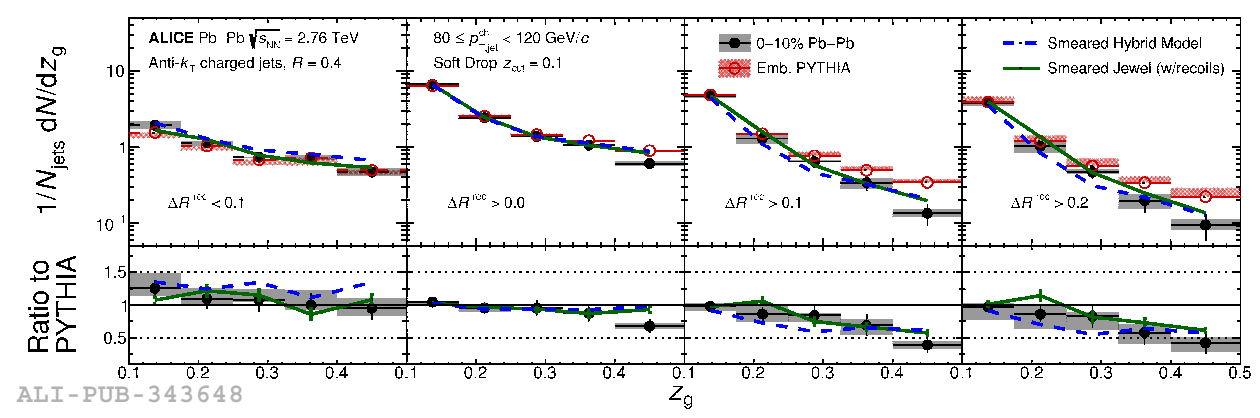

Figure 3. Raw $z_{\mathrm{g}}$ distributions as measured for $80-120 \mathrm{GeV} / \mathrm{c}$ track-based jets in $\mathrm{Pb}-\mathrm{Pb}$ collisions at $\sqrt{s_{\mathrm{NN}}}=2.76 \mathrm{TeV}$ (centrality bin $0-10 \%$ ). The measured data are compared to PYTHIA prediction which was smeared by instrumental effects and embedded into central $\mathrm{Pb}-\mathrm{Pb}$ collisions and smeared predictions of JEWEL [10] and the Hybrid model [11]. Taken from [8].

\section{Results from jet substructure measurements}

The same $\mathrm{pp}$ and $\mathrm{Pb}-\mathrm{Pb}$ data were further used to perform jet substructure measurements [8]. The analysis was carried out for track-based, anti- $k_{\mathrm{T}}$ jets with $R=0.4$ having a transverse momentum in the range $40-60 \mathrm{GeV} / c$ and $80-120 \mathrm{GeV} / c$, respectively. Figure 2 presents the fully corrected $z_{\mathrm{g}}$ and $n_{\mathrm{SD}}$ distributions measured in pp collisions at $\sqrt{s}=7 \mathrm{TeV}$. The distributions were compared with the corresponding predictions from PYTHIA simulations. All distributions were normalized by the total number of jets with transverse momentum in the range $p_{\mathrm{T}, \mathrm{jet}}^{\mathrm{ch}} \in(40,60) \mathrm{GeV} / c$, explicitly including also those jets which did not fulfill the $\mathrm{SD}$ condition $z>0.1$. PYTHIA describes the pp data well except for the the bin $n_{\mathrm{SD}}=0$ where the simulation underestimates the number of jets without a hard splitting.

In case of the central $\mathrm{Pb}-\mathrm{Pb}$ data, it was not possible to fully correct the raw $z_{\mathrm{g}}$ and $n_{\mathrm{SD}}$ distributions for smearing caused by instrumental effects and the underlying event because the unfolding turned out to be unstable due to presence of significant off-diagonal terms in the response matrix. Hence, the measured distributions were compared directly with PYTHIA calculations which were smeared by detector effects and embedded into raw $\mathrm{Pb}-\mathrm{Pb}$ events. 


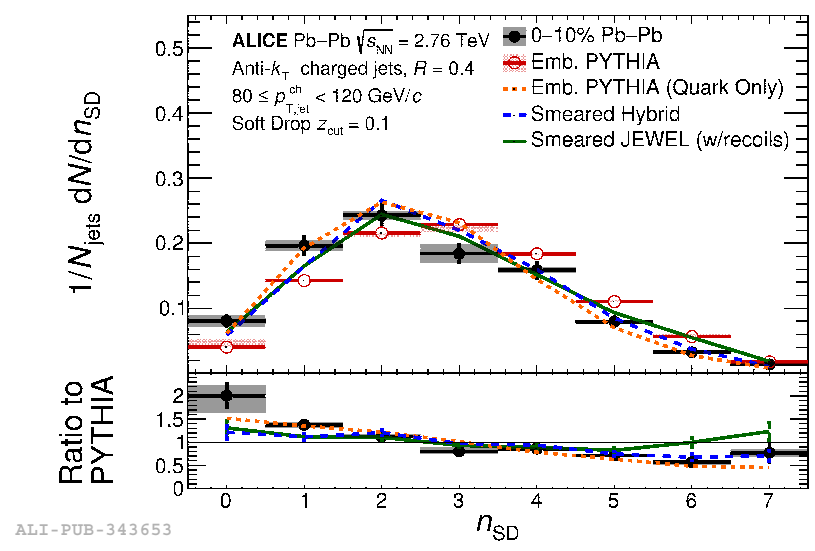

Figure 4. Raw $n_{\mathrm{SD}}$ as measured for $80-120 \mathrm{GeV} / c$ track-based jets in central $\mathrm{Pb}-\mathrm{Pb}$ collisions at $\sqrt{s_{\mathrm{NN}}}=2.76 \mathrm{TeV}$ (centrality bin $0-10 \%$ ). The measured data are compared with PYTHIA predictions which were smeared by instrumental effects and embedded into central $\mathrm{Pb}-\mathrm{Pb}$ collisions. The PYTHIA calculation was done separately for inclusive-jet and quark-jet fragmentation. The data are further compared with smeared predictions of JEWEL [10] and the Hybrid model [11]. Taken from [8].

The jet transverse momentum range $80-120 \mathrm{GeV} / c$ was chosen to be far away from the region which is dominated by combinatorial background jets. Figure 3 shows the raw measured $z_{\mathrm{g}}$ distributions separately for small and large angle splittings. The two panels on the right show that for $\mathrm{Pb}-\mathrm{Pb}$ data, splittings with a big opening angle $\left(\Delta R^{\text {rec }}>0.1\right.$ and $\left.\Delta R^{\text {rec }}>0.2\right)$, get more suppressed as the transverse momentum balance between branches in the splitting gets more symmetric, i.e. when $z_{\mathrm{g}}$ tends to 0.5 . On the other hand, this suppression is compensated by a small enhancement of small angle $\left(\Delta R^{\text {rec }}<0.1\right)$ splittings which have at the same time momentum asymmetry as it can be seen in the first panel on the left. Such behavior is qualitatively expected from the color coherence picture [9] where the medium can resolve splittings with larger opening angle as independent color charges. In addition, from angular ordering arguments, it is expected that splittings with greater opening angles occur earlier and can be thus more affected by the medium. Data are compared with JEWEL [10] and the Hybrid model [11] which both reproduce the suppression.

Figure 4 presents the corresponding raw $n_{\mathrm{SD}}$ distributions. In $\mathrm{Pb}-\mathrm{Pb}$, it can be seen that the $n_{\mathrm{SD}}$ distribution is shifted towards a lower number of splittings that pass the SD condition with respect to the embedded PYTHIA which indicates harder, more quark-like fragmentation in $\mathrm{Pb}-\mathrm{Pb}$. Thus, features of the medium induced jet shower modifications which were seen with the angularity and $p_{\mathrm{T}} D$ measurements are apparent also for the jet substructure observables.

\section{Summary}

Jet-substructure and jet-shape observables complement nuclear modification factor measurements and carry new information about the underlying mechanism of the jet quenching. The measurements presented in this paper analyzed jets having transverse momentum in the intermediate ranges $40-60 \mathrm{GeV} / c$ and $80-120 \mathrm{GeV} / c$ and jet radius $R \leq 0.4$. The data shown suggest that jet-medium interaction tends to shape these jets to have a more collimated jet core and the resulting jet fragmentation resembles the fragmentation of a quark-jet. Similar 
studies for jets with larger radii are challenging at the moment because of the large underlying event and they require new techniques to be developed.

\section{Acknowledgements}

This research was funded by the Ministry of Education, Youth, and Sports of the Czech Republic, grant number LTT17018.

\section{References}

[1] Stephanov, M.A., arXiv:hep-lat/0701002 (2006).

[2] Salam, G.P., Eur. Phys. J. C67, 637-686 (2010).

[3] Majumder, A., Van Leeuwen, M., Prog. Part. Nucl. Phys. A66, 41-92 (2011).

[4] Acharya, S. et al. (ALICE Collaboration) JHEP 10, 139 (2018).

[5] Cacciari, M., Salam, G.P. , and Soyez, G., JHEP 04, 063 (2008).

[6] Larkoski, A.J., Marzani,S., Soyez, G. and Thaler, J., JHEP 05, 146 (2014).

[7] Sjostrand,T.,Mrenna, S., and Skands, P.Z., JHEP 05, 026 (2006).

[8] ALICE Collaboration, arXiv:1905.02512v1, to be published in PLB.

[9] Casalderrey-Solana, J., Mehtar-Tani, Y., Salgado, C.A., Tywoniuk, K., Phys. Lett. B725, 357-360 (2013).

[10] Zapp,K., Ingelman, G., Rathsman,J., Stachel, J., and Wiedemann, U.A., Eur.Phys.J., C60, 617-632 (2009).

[11] Casalderrey-Solana, J., Gulhan, D.C., Milhano, J.G., Pablos, D., and Rajagopal, K., Nucl. Phys. A931, 487-492 (2014).

[12] Milhano, J.G., Wiedemann, U.A., Zapp, K.C., Phys.Lett. B779, 409-413 (2018).

[13] D’Eramo, F., Rajagopal, K., and Yin, Y., JHEP 01, 172 (2019).

[14] Mehtar-Tani, Y. and Tywoniuk, K., JHEP 04, 125 (2017).

[15] Chang,N.B., Cao, S. and Qin, G.Y., Phys.Lett. B781, 423-432 (2018). 